\title{
Diversity of carbapenem-resistant Klebsiella pneumoniae ST14 and emergence of a subgroup with KL64 capsular locus in the Arabian Peninsula
}

\author{
Shaimaa F. Mouftah ${ }^{1,2} \cdot$ Tibor Pál $^{1,3} \cdot$ Paul G. Higgins ${ }^{4,5} \cdot$ Akela Ghazawi $^{1} \cdot$ Youssef Idaghdour $^{6} \cdot$ Manaf Alqahtani $^{7}$. \\ Ali S. Omrani ${ }^{8,9} \cdot$ Tahir A. Rizvi ${ }^{1,10} \cdot$ Ágnes Sonnevend ${ }^{1,3}$ (D)
}

Received: 29 September 2021 / Accepted: 16 November 2021

(c) The Author(s) 2021

\begin{abstract}
To understand the reasons of successful spread of carbapenem-resistant Klebsiella pneumoniae ST14 (CRKP-ST14) in countries of the Arabian Peninsula, the resistome, capsular locus, carbapenemase carrying plasmid types, and core genome of isolates from the region were compared to global isolates. Thirty-nine CRKP-ST14 strains isolated from 13 hospitals in the United Arab Emirates, Bahrain, and Saudi Arabia were selected for whole genome sequencing on Illumina MiSeq platform based on the variety of carbapenemase genes carried and plasmids bearing these genes. Their resistome, capsular locus, and core genome MLST were compared to 173 CRKP-ST14 genomes available in public databases. The selected 39 CRKP-ST14 produced either NDM-1, OXA-48, OXA-162, OXA-232, KPC-2, or co-produced NDM-1 and an OXA-48like carbapenemase. cgMLST revealed three clusters: 16 isolates from five UAE cities (C1), 11 isolates from three UAE cities and Bahrain (C2), and 5 isolates from Saudi Arabia (C3), respectively, and seven singletons. Resistance gene profile, carbapenemase genes, and their plasmid types were variable in both $\mathrm{C} 1$ and $\mathrm{C} 2$ clusters. The majority of CRKP-ST14 had KL2, but members of the C2 cluster and two further singletons possessed KL64 capsular locus. Based on cgMLST comparison of regional and global isolates, CRKP-ST14 with KL64 from four continents formed a distinct cluster, suggesting a recent emergence and spread of this variant. Our findings confirmed clonal transmission coupled with likely horizontal gene transfer in carbapenem-resistant Klebsiella pneumoniae ST14. Dissemination of this genetically flexible, highly resistant clone warrants further monitoring.
\end{abstract}

Keywords Klebsiella pneumoniae $\cdot$ Carbapenem resistance $\cdot$ High-risk clone $\cdot$ Capsular switch

Shaimaa F. Mouftah and Tibor Pál contributed equally.

Ágnes Sonnevend

pal.agnes@pte.hu

1 Department of Medical Microbiology and Immunology, College of Medicine and Health Sciences, United Arab Emirates University, Al Ain, United Arab Emirates

2 Department of Biomedical Sciences, Zewail City of Science and Technology, Giza, Egypt

3 Department of Medical Microbiology and Immunology, Medical School, University of Pécs, Pécs, Hungary

4 Institute for Medical Microbiology, Immunology and Hygiene, Faculty of Medicine and University Hospital Cologne, University of Cologne, Cologne, Germany
5 German Center for Infection Research (DZIF), Partner Site Bonn-Cologne, Cologne, Germany

6 Pathology and Laboratory Medicine Department, BDF Hospital - Royal Medical Services, Riffa, Kingdom of Bahrain

7 New York University Abu Dhabi, Abu Dhabi, United Arab Emirates

8 King Faisal Specialty Hospital, Riyadh, Saudi Arabia

9 Communicable Diseases Center Hamad Medical Corporation, Doha, Qatar

10 Zayed Bin Sultan Al Nahyan Center for Health Sciences, Al Ain, United Arab Emirates 


\section{Introduction}

The emergence and spread of carbapenem-resistant Enterobacterales (CRE) is considered to be one of the most serious antibiotic resistance threats [1]. The majority of CRE is Klebsiella pneumoniae, which are carbapenem resistant due to the production of carbapenemases (CRKP) [2]. This worldwide trend is also observed in countries of the Arabian Peninsula [3-5]. Spread of CRKP is mainly driven by clonal dissemination of so called high-risk multi-drug resistant (HiR MDR) clones [2]. K. pneumoniae ST14 is one of such clones producing various carbapenemases that have been reported from four continents $[2,5,6]$ including the Arabian Peninsula [3, 7-9]. Earlier, this clone was shown to have high prevalence among CRE isolated in the United Arab Emirates $[8,10]$. To understand the reasons of its successful emergence and spread, we used whole genome sequencing (WGS) to assess the resistome, the capsular locus, the carbapenemase carrying plasmid types, as well as the level of core genome similarity of selected $K$. pneumoniae ST14 isolated in countries of the Arabian Peninsula, which were also compared to whole genomes of $K$. pneumoniae ST14 isolated at other geographical locations.

\section{Materials and methods}

\section{Identification of CRKP-ST14 isolates}

Altogether 761 independent CRKP strains isolated between June 2011 and June 2016 in 33 hospitals of five countries of the Arabian Peninsula from a collection described earlier [11] were screened by PFGE [12], to select isolates exhibiting $\geq 80 \%$ similar patterns to 23 known CRKP-ST14 identified earlier in the UAE [7-9]. These were further divided into sub-clusters based on $90 \%$ PFGE pattern similarity threshold (Supplementary Fig. 1). At least one isolate from these PFGE sub-clusters carrying unique carbapenemase genes or their combinations were subjected to multi-locus sequence typing [13] to confirm their sequence type.

\section{Testing of antibiotic susceptibility, carbapenemase production, and detection of carbapenemase genes}

Phenotypic confirmation of the isolates' carbapenemase production and methods testing their antibiotic susceptibility was described earlier in [11]. Presence of the common carbapenemase genes $\left(b l a_{\mathrm{NDM}}, b l a_{\mathrm{OXA}-48-\mathrm{like}}, b l a_{\mathrm{KPC}}\right.$, $\left.b l a_{\mathrm{VIM}}, b l a_{\mathrm{IMP}}\right)$ were screened by PCR and identified by direct sequencing of the amplicons [9].

\section{Characterization of carbapenemase gene-carrying plasmids}

Electrophoresis gels of plasmids extracted by the alkaline lysis methods from the wild-type CRKP strains were capillary transferred to Hybond $\mathrm{N}+$ membranes (Roche Diagnostics, Mannheim, Germany) and the membranes were probed with $b l a_{\mathrm{NDM}-1}$ or $b l a_{\mathrm{OXA}-48}$ PCR amplicons labelled and detected with the DIG DNA-labelling and detection kit (Roche Diagnostics, Mannheim, Germany) [14]. Selected carbapenemase bearing plasmids were conjugally transferred into a sodium-azide resistant derivative of rifampicin resistant Escherichia coli J53 (J53RAZ) [7]. If conjugation failed, $1 \mu \mathrm{g}$ of plasmid DNA purified with Plasmid Maxi Prep Kit (Qiagen, Hilden, Germany) of a wild type isolate was heatshock transformed into competent cells of Escherichia coli DH5 $\alpha$ [7]. Transfer experiments were considered successful if a single-plasmid containing derivative was obtained, confirmed by plasmid electrophoresis and PCR targeting the respective carbapenemase gene [7]. Plasmid incompatibility typing was performed by PCR as described earlier in $[3,7$, $15]$.

\section{Analysis of the whole genomes}

Genomic DNA of selected CRKP-ST14 isolates was extracted using the QIAamp DNA Mini Kit (QIAGEN, Crawley, UK), according to the manufacturer's instructions. Bacterial DNA libraries were prepared using the Illumina Nextera ${ }^{\circledR X T}$ DNA Library Preparation Kit (Illumina, USA) and sequenced on Illumina MiSeq platform using $2 \times 250 \mathrm{bp}$ or $2 \times 300 \mathrm{bp}$ paired-end sequencing technology. Reads were quality checked and assembled using the Velvet 1.1.04. algorithm built in the Ridom SeqSphere ${ }^{+}$software. Core genome MLST (cgMLST) comparison was performed using Ridom SeqSphere $^{+}$(version 7.2.3). The assembled contigs were analysed by Kleborate for the virulence gene and capsule locus related information [6]. The acquired resistance gene content was assessed using ResFinder 4.0 (https://cge.cbs. dtu.dk//services/ResFinder/) [16]. In case the Velvet assembly lacked an expected carbapenemase gene, the respective reads were assembled using SPAdes 3.11 and the contigs produced were re-analyzed in the ResFinder 4.0. The integrity of lipopolysaccharide synthesis regulating genes $\operatorname{mgr} B$, phoP, phoQ, pmrA, and $p m r B$ [17], and that of the main porin genes (ompC and $o m p F$ ) was compared to the respective genes of K. pneumoniae MGH78578 (GenBank Acc. No. CP000647). The sequence data generated were uploaded to the European Nucleotide Archives under project number PRJEB44852. 


\section{Comparison of local CRKP-ST14 isolates to ones isolated globally}

In order to compare K. pneumoniae ST14 isolates of the Arabian Peninsula genomes of three carbapenemase gene-carrying K. pneumoniae ST14 isolates: PittNDM01, KP617 and NUHL24835 (GenBank accession numbers: CP006798-CP006802, CP012753-CP012755, CP014004-CP014006) and further 170 carbapenemase carrying K. pneumoniae ST14 genomes from the PathogenWatch (https://pathogen. watch/genomes/all?genusId=570\& $\mathrm{mlst}=14 \&$ speciesId $=573$ accessed on 02-06-2021) were downloaded. All were compared by cgMLST using Ridom SeqSphere $^{+}$software. A total of 173 genomes available from the databases were also screened for their respective capsular locus types. One-hundred and nine global isolates from the databases were isolated in the same period as the recovery of our isolates from the Arabian Peninsula. Further 50 isolates used in the comparison were collected between 2017 and 2019, and 14 did not have the collection date specified.

\section{Results}

\section{Identification of Klebsiella pneumoniae ST14 isolates}

Based on PFGE, a total of $172 \mathrm{~K}$. pneumoniae strains exhibited patterns $\geq 80 \%$ similar to known CRKP-ST14 isolates. The majority of them $(n=162)$ were from hospitals in the UAE, but nine isolates from Riyadh, Saudi Arabia, and one from Riffa, Bahrain, also exhibited similar PFGE patterns. These 172 isolates could further be divided into 14 PFGE sub-clusters applying the $90 \%$ similarity threshold (Supplementary Fig. 1). All but one carried a carbapenemase gene(s), with a remarkable high rate of double carbapenemase producers (42\%). The types and distribution of carbapenemase alleles is shown in Supplementary Table 1.

Based on the PFGE subclusters and on the type of carbapenemase genes carried, 70 isolates were subjected to MLST that confirmed sequence type 14 in each case (Supplementary Fig. 1).

\section{Analysis of the carbapenemase genes and their localizations}

The 70 CRKP-ST14 isolates were further investigated by testing the plasmid localization of their respective carbapenemase genes. Based on the alleles of $b l a_{\mathrm{NDM}}$ and $b l a_{\mathrm{OXA}}$ carbapenemase genes and on their association with different plasmids, strains were divided into 13 subgroups (SGs) (Table 1). In one isolate (ABC225) no carbapenemase genes or the production of such enzymes could be detected. Two strains (ABC220 and ABC224, already described in [9]) harboured $b l a_{\mathrm{KPC}-2}$ on IncX3 plasmids, while all the others carried $b l a_{\mathrm{NDM}-1}$ alone or in combination with various alleles of $b l a_{\text {OXA-48-like }}$. The $b l a_{\text {OXA-232 }}$ gene detected by PCR could not be localized by hybridization, neither was the carbapenemase gene transferable by conjugation nor by transformation from one isolate (ABC334). On the other hand, although the plasmid electrophoresis gel of isolate ABC643 did not hybridize with the NDM-probe, from this strain the

Table 1 Subgroups of CRKP-ST14 with various alleles of carbapenemases located on different plasmids

\begin{tabular}{|c|c|c|c|c|c|c|c|c|c|c|}
\hline \multirow[t]{2}{*}{ Subgroup } & \multirow[t]{2}{*}{$N$} & \multirow{2}{*}{$\begin{array}{l}N \text { selected } \\
\text { for WGS }\end{array}$} & \multirow[t]{2}{*}{ Prototype strains* } & \multicolumn{3}{|c|}{ NDM-1 plasmid } & \multicolumn{4}{|c|}{ OXA plasmid } \\
\hline & & & & Size & Inc type & $\mathrm{C}$ & OXA allele & Size & Inc type & $\mathrm{C}$ \\
\hline A & 17 & 4 & $\mathrm{ABC} 252$ & $240 \mathrm{~Kb}$ & IncHI1B & $\mathrm{C}$ & OXA-232 & $6 \mathrm{~Kb}$ & IncColKP3 & NC \\
\hline B & 4 & 4 & SA121 & $240 \mathrm{~Kb}$ & IncHI1B & $\mathrm{C}$ & OXA-48 & $60 \mathrm{~Kb}$ & IncL & $\mathrm{C}$ \\
\hline $\mathrm{C}$ & 4 & 4 & ABC261 & $240 \mathrm{~Kb}$ & IncHI1B & $\mathrm{C}$ & OXA-162 & $60 \mathrm{~Kb}$ & IncL & $\mathrm{C}$ \\
\hline $\mathrm{D}$ & 9 & 6 & $\begin{array}{l}\text { ABC131 } \\
\text { ABC643 }\end{array}$ & $240 \mathrm{~Kb}$ & IncHI1B & $\mathrm{C}$ & none & NA & NA & NA \\
\hline $\mathrm{E}$ & 1 & 1 & $\mathrm{ABC} 240$ & $120 \mathrm{~Kb}$ & IncFII & $\mathrm{C}$ & OXA-232 & $60 \mathrm{~Kb}$ & IncColKP3, IncR & $\mathrm{NC}$ \\
\hline $\mathrm{F}$ & 7 & 4 & RH60 & $120 \mathrm{~Kb}$ & IncFII & $\mathrm{C}$ & OXA-232 & $6 \mathrm{~Kb}$ & IncColKP3 & $\mathrm{NC}$ \\
\hline G & 3 & 3 & DHKp6 & $120 \mathrm{~Kb}$ & IncFII & $\mathrm{C}$ & none & NA & NA & NA \\
\hline $\mathrm{H}$ & 5 & 5 & MCH15 & NA & NA & NA & OXA-162 & $60 \mathrm{~Kb}$ & IncL & $\mathrm{NC}$ \\
\hline I & 1 & 1 & $\mathrm{ABC} 232$ & NA & NA & NA & OXA-232 & $10 \mathrm{~Kb}$ & IncColKP3 & $\mathrm{NC}$ \\
\hline $\mathrm{J}$ & 15 & 4 & $\mathrm{ABC} 270$ & NA & NA & NA & OXA-232 & $6 \mathrm{~Kb}$ & IncColKP3 & NC \\
\hline K & 1 & 1 & $\mathrm{ABC} 334$ & NA & NA & NA & OXA-232 & not loca & ybridization & NC \\
\hline $\mathrm{L}$ & 2 & 1 & $\mathrm{ABC} 220$ & \multicolumn{7}{|c|}{ KPC-2 on IncX3 plasmid } \\
\hline M & 1 & 1 & $\mathrm{ABC} 225$ & \multicolumn{7}{|c|}{ Carbapenemase negative } \\
\hline
\end{tabular}

*Strain from which the plasmid transfer and incompatibility typing was performed

$C$ conjugative, $N C$ non-conjugative, $N A$ not applicable 
$b l a_{\mathrm{NDM}-1}$ was successfully transferred by conjugation into $E$. coli $\mathrm{J} 53_{\mathrm{RAZ}}$ and the carbapenemase gene was identified on an approx. $240 \mathrm{~kb}$ IncHI1B plasmid from the transconjugant. Consequently, ABC643 was considered being member of subgroup D (Table 1). From each of the 13 subgroups identified, up to five isolates, overall 39 isolates were selected for whole genome sequencing (Table 2).

\section{Clonal relatedness and resistome of the carbapenem-resistant K. pneumoniae ST14}

The assembly statistics of the 39 isolates subjected to WGS is summarized in Supplementary Table 2. The cgST types are listed in Table 2. Core genome MLST revealed seven singletons and three clusters that differed by up to 14 out of 2358 alleles (Table 2 and Fig. 1).

As it is shown in Table 2, Cluster 1 and Cluster 2 contained isolates from various hospitals in different cities carrying different carbapenemases, whereas Cluster 3 consisted of isolates exclusively from one hospital in Saudi Arabia.

Beyond the carbapenemase and other beta-lactamase genes, all isolates carried acquired resistance genes coding for aminoglycoside, trimethoprim and sulfamethoxazole resistance, and they variably possessed macrolide, tetracycline, rifampicin and plasmid mediated quinolone resistance genes. Importantly, the majority of isolates in the three clusters carried the armA $16 \mathrm{~S}$ methylase gene. The array of acquired antibiotic resistance genes matched the antimicrobial susceptibility profiles of the isolates (Supplementary Table 3), e.g., high-level tetracycline resistance was observed with isolates possessing the $\operatorname{tet}(D)$ gene. Similarly, the few isolates susceptible to chloramphenicol were the ones lacking any phenicol resistance genes. However, the lack of acquired phenicol resistance genes did not translate always to susceptibility to chloramphenicol, suggesting alternative resistance mechanisms, possibly efflux. The colistin resistance observed was not due to the presence of mobile colistin resistance genes ( $m c r 1-9$ ) and did not correlate with certain clusters. Colistin resistance in 7 of the 13 colistin resistant isolates was due to insertional, frameshift or non-synonymous point mutations of the $m g r B$ gene. In further two isolates point mutations in $p h o Q$, and in another three point mutations in $\mathrm{pmrB}$ were responsible for this phenotype. In one isolate the regulatory genes examined were all wild-type (Table 2), but further investigation of the colistin resistance mechanism in this single isolate was beyond the scope of our current study.

We assume that the only carbapenemase non-producing strain $(\mathrm{ABC} 225)$ was resistant to carbapenems due to the production of multiple beta-lactamases, including ones with extended-spectrum $\left(b l a_{\mathrm{TEM}-1 \mathrm{~A}}, b l a_{\mathrm{OXA}-1}, b l a_{\mathrm{OXA}-9}\right.$, $\left.b l a_{\text {CTX-M-15 }}\right)$ combined with deletions in the ompF gene: a 19 base-long one starting at nucleotide 676 and a single nucleotide one at position 872 , while the $\operatorname{ompC}$ gene was wild-type (data not shown).

All isolates, but $\mathrm{ABC} 681$ possessed the yersiniabactin siderophore genes encoded on ICEKp 5 mobile element, but none of them had genes characteristic to hypervirulent K. pneumoniae, i.e., rmpA, rmpA2, and iutA. Interestingly, isolates in Cluster 2 and further two singletons (RH60 and

Table 2 Characteristics, cgMLST clustering, and resistome of strains subjected to whole genome sequencing

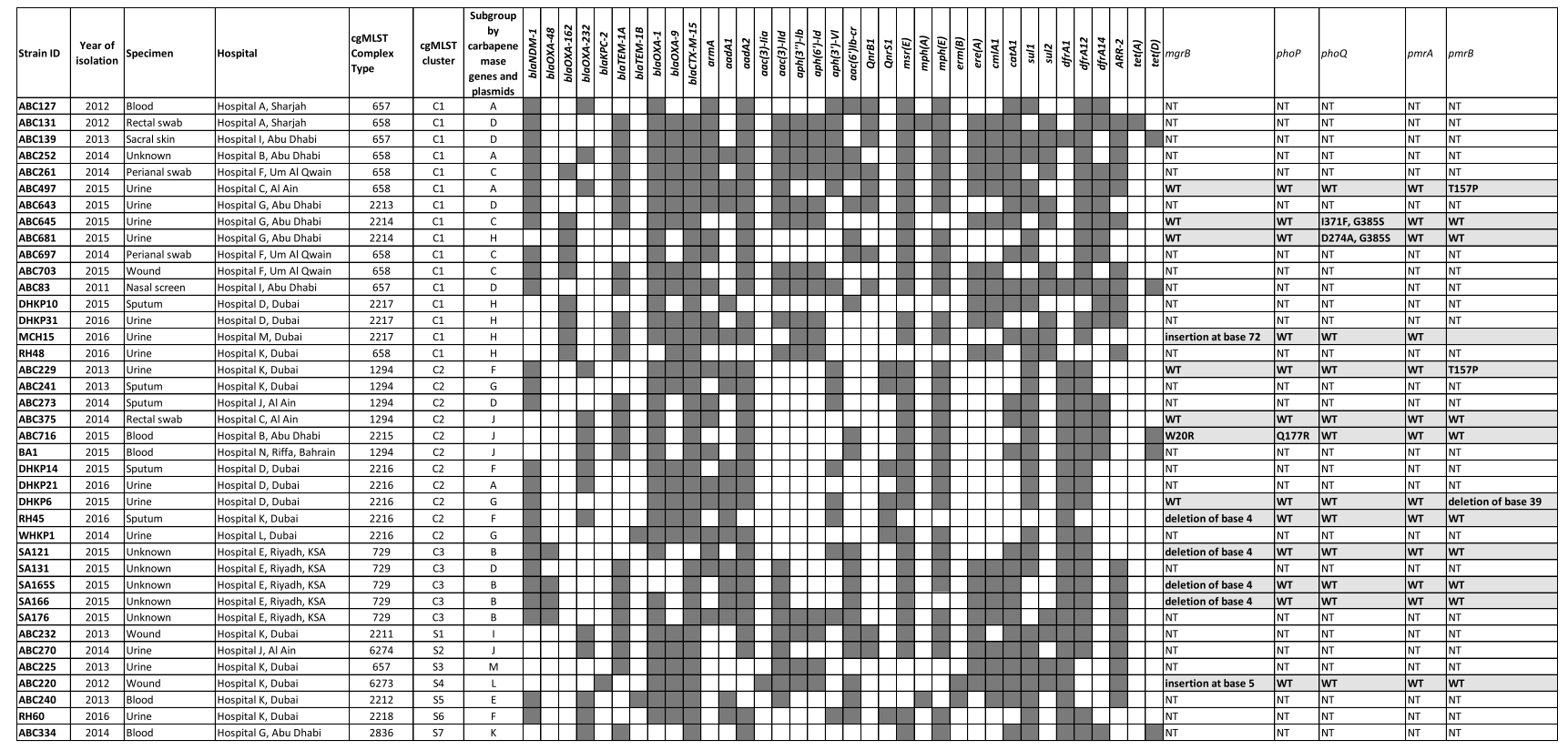

$W T$ wild type, $N T$ not tested 
Fig. 1 Minimum spanning tree based on cgMLST of the $39 \mathrm{~K}$. pneumoniae ST14. Distance is based on number of differences of the 2358 alleles in $K$. pneumoniae sensu lato cgMLST scheme with cluster alert distance set at 15

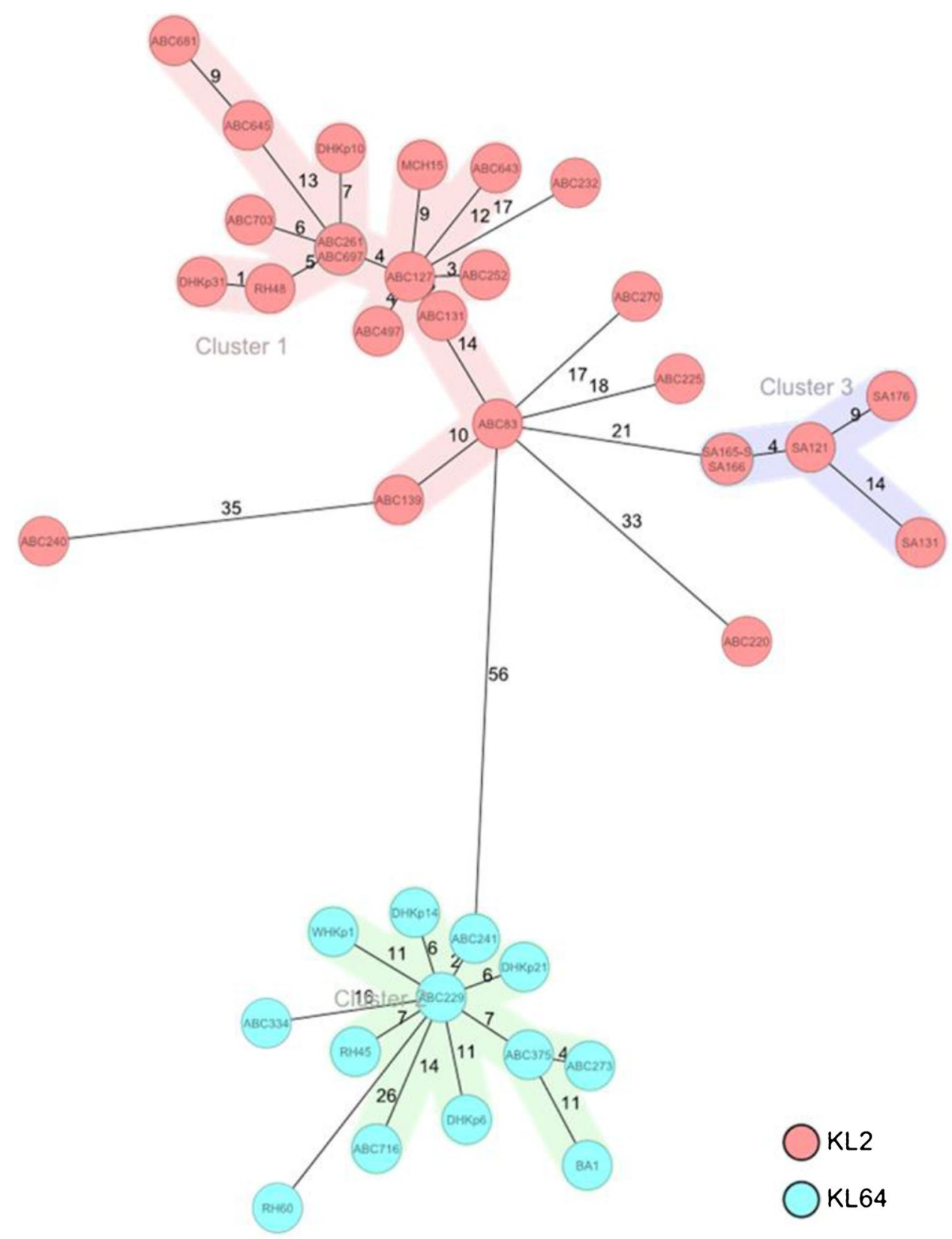

ABC334) possessed capsular locus KL64, not the KL2, i.e., one typically associated with $K$. pneumoniae ST14 and also present in the remaining isolates (Fig. 1). Although isolates carrying the KL64 locus clustered by their core genome, the carbapenemase genes carried by them were variable: some of them possessed $b l a_{\mathrm{NDM}-1}$, others $b l a_{\mathrm{OXA}-232}$, or both of these carbapenemases (Table 2 and Fig. 2).

\section{Comparison of K. pneumoniae ST14 isolated in the Arabian Peninsula to international K. pneumoniae ST14 genomes}

The neighbour-joining tree generated by comparing the cgMLST of local as well as global, altogether $212 \mathrm{~K}$. pneumoniae ST14 isolates is presented in Fig. 2. K. pneumoniae 

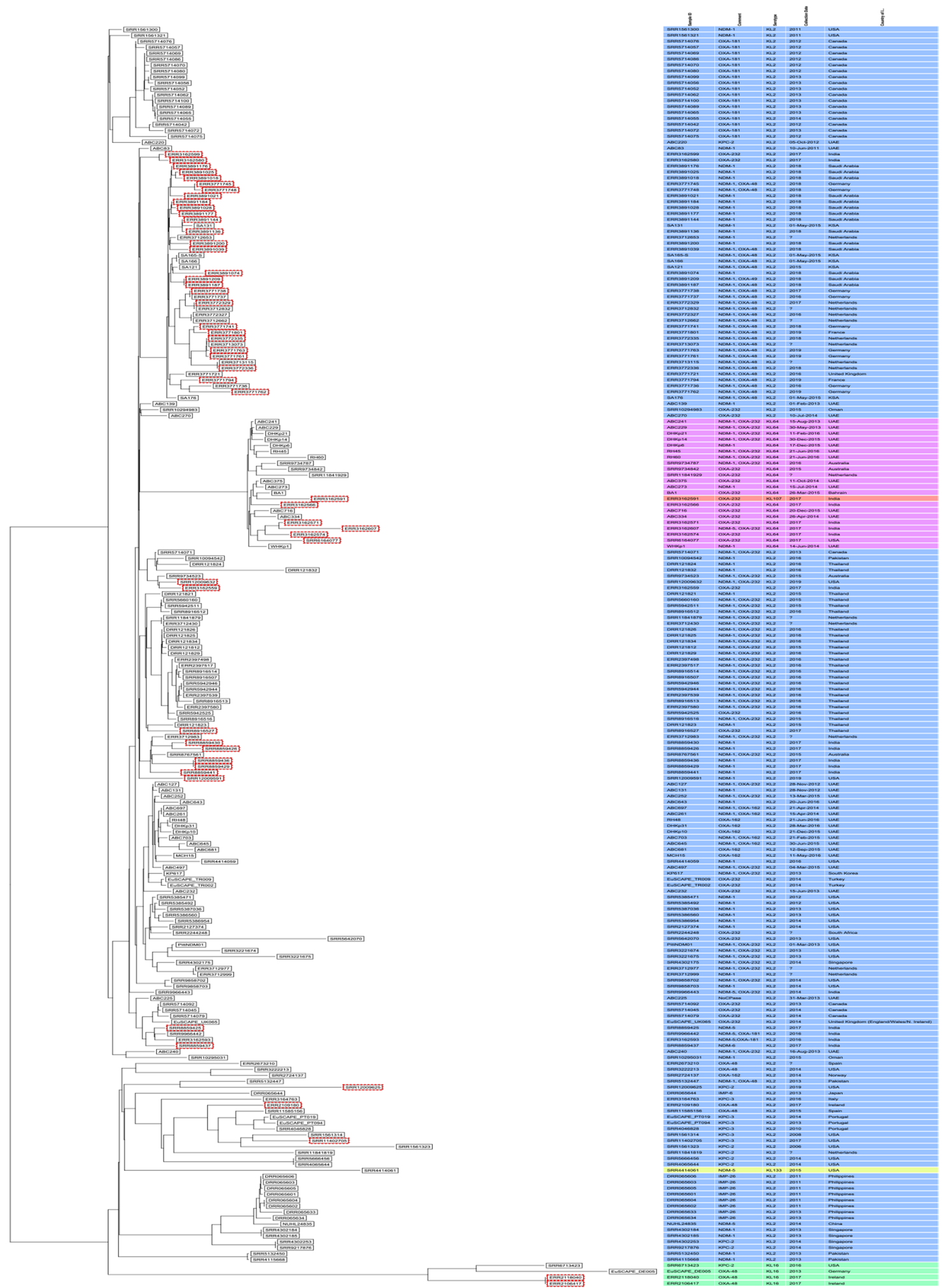

KL107

OKL133

OKL16

OKL2

OKL64

m 
4Fig. 2 Neighbour-joining tree based on the cgMLST of 212 K. pneumoniae ST14. Color code is based on the capsular locus type. The strains marked with red dotted line are isolated in 2017-2019, i.e., after the strains investigated in the present study

ST14 genomes with KL2 capsular locus formed several clusters, whereas isolates of the ST14 clone having other capsular locus types (KL16, KL64, KL107, and KL133) clearly separated from the previous groups. The core genomes of $K$. pneumoniae ST14 harbouring KL64 from the UAE and Bahrain formed a distinct cluster with NDM-1 or NDM-5 and/or OXA-232 producing K. pneumoniae ST14 isolates from the Netherlands, Australia, India and the USA and with one isolate from India with capsular locus KL107, which also contained the wzi64 allele.

\section{Discussion}

Numerous high-risk $K$. pneumoniae clones, among them ST14, have now been identified [2]. ST14 strains have been reported to produce a wide range of carbapenemases: apart from being the sequence type from which the $b l a_{\mathrm{NDM}-1}$ gene was first identified [18], various $b a_{\mathrm{KPC}}[9,19,20]$, bla $_{\mathrm{NDM}}$ [21, 22], and bla OXA-48-like $[6,23-26]$ alleles or double carbapenemase producers have been reported from different parts of the world, including the Middle East [7, 8, 27-31]. Furthermore, ST14 strains appear to be one of the most wide-spread, highly resistant CRE clones in Dubai [8].

Even in the light of this variety of CRKP-ST14 strains, the diversity of carbapenem-resistant mechanisms encountered in the relatively small pool of ST14 strains isolated in countries of the Arabian Peninsula was remarkable (Table 1). Beyond strains carrying $b l a_{\mathrm{NDM}-1}, b l a_{\mathrm{OXA}-232}, b l a_{\mathrm{KPC}-2}$, and combinations of $b l a_{\mathrm{NDM}-1}$ either with $b l a_{\mathrm{OXA}-232}$, or with $b l a_{\text {OXA-48, }}$, we identified isolates with $b l a_{\text {OXA-162. }}$. To the best of our knowledge, this is the first observation of this allele alone or in combination with $b l a_{\mathrm{NDM}-1}$ in this high-risk clone of $K$. pneumoniae. This variety of carbapenemase gene content further confirms the earlier observations describing this high-risk clone as one which is particularly fit to acquire resistance plasmids and spread the resistance traits $[2,6]$. Furthermore, we also encountered a strain, which did not produce a dedicated carbapenemase, yet was resistant to carbapenems due to ESBL production and $o m p F$ mutation, a phenomenon otherwise not uncommon among CRKP [10, $11]$.

The diversity of ST14 strains was further accentuated by the various types of, mostly conjugative, plasmids carrying the carbapenamase genes. Even the same allele of a carbapenemase gene $\left(b l a_{\mathrm{NDM}-1}\right)$ could be localised on two different plasmids, IncHIIb and IncFII, in our isolates. On the other hand, all $b l a_{\text {OXA- } 48}$ and $b l a_{\text {OXA-162 }}$ were located on
IncL type plasmids, while $b l a_{\mathrm{OXA}-232}$ was associated with IncColKP 3 type plasmids and in a single isolate (ABC240) with an IncColKP3-IncR composite plasmid (Table 1).

The cgMLST results did not completely overlap with these carbapenemase gene-, and plasmid-based groups (Table 1 and 2). Considering only the carbapenemase genes, members of cgMLST cluster 3 were the most homogenous, as all such strains produced NDM and OXA- 48 with the exception of a single isolate, presumably having lost the plasmid coding for the latter carbapenemase. This lack of heterogeneity was not surprising as all these strains were isolated in the same hospital in Saudi Arabia. Interestingly, they clustered with Saudi isolates of 2018, and German isolates of 2016-2019 deposited in the PathogenWatch database (Fig. 2). On the other hand, members of the other two cgMLST clusters were more heterogeneous: despite being highly similar at the genome level: they carried a variety of plasmids coding for a variety of carbapenemase alleles coded on different plasmids. In some cases, such differences were even seen among isolates from the same hospital (e.g., see Cluster C1 isolates from Hospital G in Abu Dhabi, Table 2). This considerable heterogeneity within the same ST, even within the same cgMLST cluster, suggests that horizontal transfer of carbapenemase gene-coding plasmids considerably contributes to the epidemiology of ST14 even in a relatively small region. Nevertheless, the fact that same cgMLST group strains possessing the same carbapenemase bearing plasmid could be isolated from multiple hospitals (e.g., Cluster $\mathrm{C} 1$ strains of plasmid group A from hospitals $\mathrm{A}$ in Sharjah, $\mathrm{C}$ in Al Ain, and B in Abu Dhabi) indicates the importance of clonal, inter-hospital spread, as well.

The variety and wide array of genes coding for resistance to numerous antibiotic classes within clusters were also notable findings supporting the previous notion. It also explains how members of the clone develop to being extremely drug resistant, or even pan-drug resistant, which was already observed in the region $[8,30]$.

Recently, and concerningly the convergent evolution of antimicrobial resistance and virulence was observed in K. pneumoniae, mostly, but not exclusively, in SouthEast Asia [6, 32, 33]. Some of these hypervirulent isolates expressed non-K1 capsular types, i.e., K2, K54, or K64 [33, 34]. Although virulence genes typically associated with hypervirulent $K$. pneumoniae (rmpA/A2, iutA) were not identified in our strains (data not shown), the two capsular types (KL2 and KL64) detected in carbapenemresistant $K$. pneumoniae ST14 have commonly been linked to hypervirulence and invasive diseases $[6,33,35]$. Further studies are needed to investigate whether this capsular switch in CRKP-ST14 from the well-known K2 type to K64 increases or changes virulence, or simply helps the pathogen to avoid immune recognition, and helps the dissemination of this clone, as was hypothesized in case of 
KPC-producing $K$. pneumoniae $\mathrm{CC} 258$ with capsular variations $[35,36]$. Our data does not allow us to speculate on the molecular mechanisms involved in the emergence of K64 strains. Nevertheless, as the K2 and K64 loci are considerably different [37], the role of recombination rather than local, mutation-based evolution seems to be more likely. However, irrespective of the mechanism behind, the clustering of cgMLST of ST14-K64 isolates collected in 2013-2016 from the UAE and Bahrain and those of other three continents, i.e., Australia from 2016, USA and India from 2017, and the Netherlands (Fig. 2) suggests a potentially emerging subgroup of the K. pneumoniae ST14 clone. The dissemination and virulence potential of this subgroup needs further monitoring.

Supplementary Information The online version contains supplementary material available at https://doi.org/10.1007/s10096-021-04384-2.

Author contribution SFM carried out molecular typing, plasmid analysis, detection of carbapenemase genes, participated in whole genome sequencing, in data analysis and in drafting the manuscript, TP participated in designing the study, in the collection of the isolates, in data interpretation and in drafting the manuscript, PGH participated in whole genome sequencing, in data analysis, and in drafting the manuscript, AG participated in molecular typing by PFGE and MLST, and in drafting the manuscript, YI participated in whole genome sequencing and in data analysis, MA and ASO participated in collecting the isolates and in drafting the manuscript, TAR participated in molecular testing and in drafting the manuscript, and AS initiated the study, participated in its design, in the analysis of all data, in drafting the manuscript.

Funding Open access funding provided by University of Pécs. This work was supported by grants UAEU UPAR-31M235 and Kispál Gyula Grant of The Pécs University Medical School 300852 awarded to AS.

Data availability The sequence data generated were uploaded to the European Nucleotide Archives under project number PRJEB44852.

Code availability Not applicable.

\section{Declarations}

Ethics approval Ethics approval was not necessary as no samples have been collected for the sake of this particular study, the information was recorded in a manner that subjects could not be identified and there were no personal identifiers linked to the specimens and the project did not involve identifiable living humans.

Consent to participate Not applicable.

Consent to publication All co-authors agreed to the submission of the current form of the manuscript.

Conflict of interest TP has received grants independently of this project, from Merck, Pfizer and Becton-Dickinson. The other authors have no conflict of interest to declare.

Open Access This article is licensed under a Creative Commons Attribution 4.0 International License, which permits use, sharing, adaptation, distribution and reproduction in any medium or format, as long as you give appropriate credit to the original author(s) and the source, provide a link to the Creative Commons licence, and indicate if changes were made. The images or other third party material in this article are included in the article's Creative Commons licence, unless indicated otherwise in a credit line to the material. If material is not included in the article's Creative Commons licence and your intended use is not permitted by statutory regulation or exceeds the permitted use, you will need to obtain permission directly from the copyright holder. To view a copy of this licence, visit http://creativecommons. org/licenses/by/4.0/.

\section{References}

1. Tacconelli E, Carrara E, Savoldi A, Harbarth S, Mendelson M, Monnet DL, Pulcini C, Kahlmeter G, Kluytmans J, Carmeli Y, Ouellette M, Outterson K, Patel J, Cavaleri M, Cox EM, Houchens CR, Grayson ML, Hansen P, Singh N, Theuretzbacher U, Magrini N, WHO Pathogens Priority List Working Group (2018) Discovery, research, and development of new antibiotics: the WHO priority list of antibiotic-resistant bacteria and tuberculosis. Lancet Infect Dis 18:318-327. https://doi.org/10.1016/S1473-3099(17) 30753-3

2. Navon-Venezia S, Kondratyeva K, Carattoli A (2017) Klebsiella pneumoniae: a major worldwide source and shuttle for antibiotic resistance. FEMS Microbiol Rev 41:252-275

3. Dortet L, Poirel L, Al Yaqoubi F, Nordmann P (2012) NDM1, OXA-48 and OXA-181 carbapenemase-producing Enterobacteriaceae in Sultanate of Oman. Clin Microbiol Infect 18:E144-E148

4. Al-Abdely H, AlHababi R, Dada HM, Roushdy H, Alanazi MM, Alessa AA et al (2021) Molecular characterization of carbapenem-resistant Enterobacterales in thirteen tertiary care hospitals in Saudi Arabia. Ann Saudi Med 41:63-70

5. Wu W, Feng Y, Tang G, Qiao F, McNally A, Zong Z (2019) NDM metallo- $\beta$-lactamases and their bacterial producers in health care settings. Clin Microbiol Rev 32:e00115-e118

6. Wyres KL, Nguyen TNT, Lam MMC, Judd LM, van Vinh CN, Dance DAB et al (2020) Genomic surveillance for hypervirulence and multi-drug resistance in invasive Klebsiella pneumoniae from South and Southeast Asia. Genome Med 12:11. https://doi.org/10. 1186/s13073-019-0706-y

7. Al-Baloushi AE, Pál T, Ghazawi A, Sonnevend A (2018) Genetic support of carbapenemases in double carbapenemase producer Klebsiella pneumoniae isolated in the Arabian Peninsula. Acta Microbiol Immunol Hung 65:135-150

8. Moubareck CA, Mouftah SF, Pál T, Ghazawi A, Halat DH, Nabi A et al (2018) Clonal emergence of Klebsiella pneumoniae ST14 co-producing OXA-48-type and NDM carbapenemases with high rate of colistin resistance in Dubai, United Arab Emirates. Int J Antimicrob Agents 52:90-95

9. Sonnevend A, Ghazawi A, Darwish D, AlDeesi Z, Kadhum AFAF, Pál T et al (2014) Characterization of KPC-type carbapenemaseproducing Klebsiella pneumoniae strains isolated in the Arabian Peninsula. J Antimicrob Chemother 70:1592-1593

10. Sonnevend Á, Ghazawi AA, Hashmey R, Jamal W, Rotimi VO, Shibl AM et al (2015) Characterization of carbapenem-resistant Enterobacteriaceae with high rate of autochthonous transmission in the Arabian Peninsula. PLoS One 10:e0131372

11. Sonnevend Á, Ghazawi A, Darwish D, Barathan G, Hashmey R, Ashraf $T$ et al (2020) In vitro efficacy of ceftazidime-avibactam, aztreonam-avibactam and other rescue antibiotics against carbapenem-resistant Enterobacterales from the Arabian Peninsula. Int J Infect Dis 99:253-259 
12. Gautom RK (1997) Rapid pulsed-field gel electrophoresis protocol for typing of Escherichia coli O157:H7 and other gram-negative organisms in 1 day. J Clin Microbiol 35:2977-2980

13. Diancourt L, Passet V, Verhoef J, Grimont PAD, Brisse S (2005) Multilocus sequence typing of Klebsiella pneumoniae nosocomial isolates. J Clin Microbiol 43:4178-4182

14. Sonnevend A, Al Baloushi A, Ghazawi A, Hashmey R, Girgis S, Hamadeh MB et al (2013) Emergence and spread of NDM-1 producer Enterobacteriaceae with contribution of IncX3 plasmids in the United Arab Emirates. J Med Microbiol 62:1044-1050

15. Poirel L, Bonnin RA, Nordmann P (2012) Genetic Features of the Widespread Plasmid Coding for the Carbapenemase OXA-48. Antimicrob Agent Chemother 56:559-562

16. Zankari E, Hasman H, Cosentino S, Vestergaard M, Rasmussen $\mathrm{S}$, Lund $\mathrm{O}$ et al (2012) Identification of acquired antimicrobial resistance genes. J Antimicrob Chemother 67:2640-2644

17. Olaitan AO, Morand S, Rolain JM (2014) Mechanisms of polymyxin resistance: acquired and intrinsic resistance in bacteria. Front Microbiol 26(5):643. https://doi.org/10.3389/fmicb.2014. 00643

18. Yong D, Toleman MA, Giske CG, Cho HS, Sundman K, Lee K et al (2009) Characterization of a new metallo- $\beta$-lactamase gene, $b l a_{\mathrm{NDM}-1}$, and a novel erythromycin esterase gene carried on a unique genetic structure in Klebsiella pneumoniae sequence type 14 from India. Antimicrob Agents Chemother 53:5046-5054

19. Ocampo AM, Chen L, Cienfuegos AV, Roncancio G, Chavda $\mathrm{KD}$, Kreiswirth BN et al (2016) A two-year surveillance in five Colombian tertiary care hospitals reveals high frequency of nonCG258 clones of carbapenem-resistant Klebsiella pneumoniae with distinct clinical characteristics. Antimicrob Agents Chemother 60:332-342

20. Krapp F, Morris AR, Ozer EA, Hauser AR (2017) Virulence characteristics of carbapenem-resistant Klebsiella pneumoniae strains from patients with necrotizing skin and soft tissue infections. Sci Rep 7. https://doi.org/10.1038/s41598-017-13524-8

21. Giske CG, Fröding I, Hasan CM, Turlej-Rogacka A, Toleman M, Livermore D et al (2012) Diverse sequence types of Klebsiella pneumoniae contribute to the dissemination of bla NDM-1 in India, Sweden, and the United Kingdom. Antimicrob Agents Chemother 56:2735-2738

22. Mei YF, Liu PP, Wan LG, Liu Y, Wang LH, Wei DD et al (2017) Virulence and genomic feature of a Virulent Klebsiella pneumoniae sequence type 14 strain of serotype K2 harboring blaNDM-5 in China. Front Microbiol 8. https://doi.org/10.3389/fmicb.2017. 00335

23. Piccirilli A, Perilli M, Piccirilli V, Segatore B, Amicosante G, Maccacaro L et al (2020) Molecular characterization of carbapenem-resistant Klebsiella pneumoniae ST14 and ST512 causing bloodstream infections in ICU and surgery wards of a tertiary university hospital of Verona (northern Italy): co-production of KPC-3, OXA-48, and CTX-M-15 $\beta$-la. Diagn Microbiol Infect Dis 96:114968

24. Grundmann H, Glasner C, Albiger B, Aanensen DM, Tomlinson CT, Andrasević AT et al (2017) Occurrence of carbapenemaseproducing Klebsiella pneumoniae and Escherichia coli in the European survey of carbapenemase-producing Enterobacteriaceae (EuSCAPE): a prospective, multinational study. Lancet Infect Dis 17:153-163

25. Österblad M, Kirveskari J, Hakanen AJ, Tissari P, Vaara M, Jalava J (2012) Carbapenemase-producing Enterobacteriaceae in Finland: the first years (2008-11). J Antimicrob Chemother 67:2860-2864
26. Jayol A, Poirel L, Brink A, Villegas MV, Yilmaz M, Nordmann P (2014) Resistance to colistin associated with a single amino acid change in protein PmrB among Klebsiella pneumoniae isolates of worldwide origin. Antimicrob Agents Chemother 58:4762-4766

27. Doi Y, Hazen TH, Boitano M, Tsai YC, Clark TA, Korlach J et al (2014) Whole-genome assembly of Klebsiella pneumoniae coproducing NDM-1 and OXA-232 carbapenemases using singlemolecule, real-time sequencing. Antimicrob Agents Chemother 58:5947-5953

28. Shankar C, Venkatesan M, Rajan R, Mani D, Lal B, Antony J et al (2019) Molecular characterization of colistin-resistant Klebsiella pneumoniae \& its clonal relationship among Indian isolates. Indian J Med Res 149:199-207. https://doi.org/10.4103/ijmr. IJMR_2087_17

29. Lomonaco S, Crawford MA, Lascols C, Timme RE, Anderson K, Hodge DR et al (2018) Resistome of carbapenem- and colistin-resistant Klebsiella pneumoniae clinical isolates. PLoS One 13:e0198526

30. Alghoribi MF, Alqurashi M, Okdah L, Alalwan B, AlHebaishi YS, Almalki A et al (2021) Successful treatment of infective endocarditis due to pandrug-resistant Klebsiella pneumoniae with ceftazidime-avibactam and aztreonam. Sci Rep 11:9684. https:// doi.org/10.1038/s41598-021-89255-8

31. Naha S, Sands K, Mukherjee S, Saha B, Dutta S, Basu S (2021) OXA-181-like carbapenemases in Klebsiella pneumoniae ST14, ST15, ST23, ST48, and ST231 from septicemic neonates: coexistence with NDM-5, resistome, transmissibility, and genome diversity. MSphere 6:e01156-e1220. https://doi.org/10.1128/mSphere. 01156-20

32. Yang Q, Jia X, Zhou M, Zhang H, Yang W, Kudinha T et al (2020) Emergence of ST11-K47 and ST11-K64 hypervirulent carbapenem-resistant Klebsiella pneumoniae in bacterial liver abscesses from China: a molecular, biological, and epidemiological study. Emerg Microbes Infect 9:320-331

33. Turton JF, Payne Z, Coward A, Hopkins KL, Turton JA, Doumith $\mathrm{M}$ et al (2018) Virulence genes in isolates of Klebsiella pneumoniae from the UK during 2016, including among carbapenemase gene-positive hypervirulent K1-ST23 and 'non-hypervirulent' types ST147, ST15 and ST383. J Med Microbiol 67:118-128

34. Zhang Y, Jin L, Ouyang P, Wang Q, Wang R, Wang J et al (2020) Evolution of hypervirulence in carbapenem-resistant Klebsiella pneumoniae in China: a multicentre, molecular epidemiological analysis. J Antimicrob Chemother 75:327-336

35. Wick RR, Heinz E, Holt KE, Wyres KL (2018) Kaptive web: user-friendly capsule and lipopolysaccharide serotype prediction for Klebsiella genomes. J Clin Microbiol 56:197-215

36. Wyres KL, Gorrie C, Edwards DJ, Wertheim HF, Hsu LY, VanKKinh $\mathrm{N}$ et al (2015) Extensive capsule locus variation and largescale genomic recombination within the Klebsiella pneumoniae clonal group 258. Genome Biol Evol 7:1267-1279

37. Pan Y, Lin T, Chen C, Chen Y, Hsieh P, Hsu C et al (2015) Genetic analysis of capsular polysaccharide synthesis gene clusters in 79 capsular types of Klebsiella spp. Sci Rep 5:15573. https://doi.org/ $10.1038 /$ srep 15573

Publisher's Note Springer Nature remains neutral with regard to jurisdictional claims in published maps and institutional affiliations. 